\title{
Fitness in Contemporary Dance: A Systematic Review
}

\author{
Authors \\ M. Angioi ${ }^{1}$, G. Metsios ${ }^{1,2}$, Y. Koutedakis ${ }^{1,2,3}$, M. A. Wyon \\ Affiliations \\ ${ }^{1}$ School of Sport, Performing Arts and Leisure, University of Wolverhamtpon, Walsall, United Kingdom \\ ${ }^{2}$ Institute of Human Performance and Rehabilitation, CERETETH, Trikala, Greece \\ ${ }^{3}$ Department of Exercise Sciences, University of Thessaly, Trikala, Greece
}

\section{Key words \\ - dancers \\ - aerobic \\ - anaerobic \\ - muscular strength \\ - body composition}

accepted after revision January 18, 2009

Bibliography DOI 10.1055/s-0029-1202821

Published online:

March 19, 2009

Int J Sports Med 2009; 30:

475-484 @ Georg Thieme

Verlag KG Stuttgart · New York ISSN 0172-4622

\section{Correspondence \\ M. Angioi, MSc, PhD candidate}

School of Sport, Performing

Arts and Leisure

University of Wolverhamtpon

Gorway Road

Walsall

United Kingdom

WS1 3BD

Tel.: +44/7789/12 7883

Fax: +44/1902/32 2894

m.angioi@wlv.ac.uk

\section{Abstract}

\section{$\nabla$}

has been suggested that dancers are less fit compared to other athletes. However, the majority of studies make their arguments based on data deriving mainly from ballet. Therefore, the aim of the current review was to investigate: a) aerobic and anaerobic fitness, muscular strength and body composition characteristics in contemporary dancers of different levels, and b) whether supplementary exercise interventions, in addition to normal dance training, further improves contemporary dance performance. Three databases (Medline, Cochrane and the Cumulative Index to Nursing \& Allied Health research database) were searched to identify publications

\section{Introduction}

$\nabla$

Contemporary (or modern) dance has emerged in the last century. As form of expression dance can be traced as long back to human culture and history as ancient Egypt and Greece. The muscle's ability to convert chemical energy from food into muscular work is directly related to performance in dance and sport alike. Moreover, similar to other sports, dance performance depends on a large number of technical, medical, psychological, nutritional, economic, environmental and physiological elements. At professional level, for instance, dancers must be experts in the aesthetic and technical side of the art, psychologically prepared to handle the stress of critical situations and be free from injury; most importantly they must be physically 'fit' [28].

The last three decades have witnessed an unprecedented exercise and fitness- "boom", reflected in the large number of people engaged in some forms of physical activity. Never before has so much capital and effort been invested in an attempt to maintain/improve physical fitness and regarding the main fitness components of contemporary professional and student dancers. At a professional level, it appears that contemporary dancers demonstrate higher maximal oxygen uptake and higher scores in muscular endurance than ballet dancers. However, contemporary dance students are equally fit compared to their ballet counterparts and their body composition is also very similar. Only two studies have investigated the effects of supplementary exercise training on aspects of dance performance. Further research is needed in order to confirm preliminary data, which suggest that the implementation of additional fitness training is beneficial for contemporary dance students to achieve a better performance outcome.

increase performance both in the exercise field and the working environment. Physical fitness may be defined as "the individuals' ability to meet the demands of a specific physical task"[26], and primarily consists of aspects related to muscle and its function. It depends on the individuals' ability to work under aerobic and anaerobic conditions, and on their capacity to develop high levels of muscular tension (or strength); muscular power, joint mobility, muscle flexibility and body composition are also equally important components of physical fitness [18].

The physical demands placed on dancers from current choreography and performance schedules make their physiology and fitness just as important as skill development. As a result, they have been referred to as "performing" [26] and/or "aesthetic" [53] athletes who remain subject to the same unyielding physical laws as in other athletes. It has been suggested that there are two main physiological requirements necessary for dancers [26]: one is a large reserve of power, required for explosive jumps and high elevation, which lasts just a few seconds, energised by 
phosphocreatine; and the other requirement suggested is muscular endurance, and occurs when a relatively high power output is maintained for 30-60 seconds. This could be, for example, in a series of jumps. Available data also suggest that dance performance benefits from enhanced physiological capabilities such as muscular strength and power $[11,25]$, in line with other aesthetic sports, such as gymnastics $[1,19]$. Since contemporary dance is predominantly an intermittent type of exercise [52], similar to soccer or tennis where explosive bursts of action are followed by moments requiring precision and skill, dancers would further benefit from a good aerobic foundation [2], while a high anaerobic threshold would limit the deleterious effects of metabolite accumulation in activities requiring balance, poise and co-ordination [6]. However, although dance involves several hours of daily practice, published data reveal that dancers have reduced fitness levels compared to athletes from other sports [6].

When studies report that dancers are less fit compared to other athletes, they make their arguments based on data deriving mainly from ballet $[22,26,56]$. Moreover, when referred to dancers, authors rarely make a differentiation regarding the levels of dancers (i.e. student and professional); such differentiation may be important since non professional and professional athletes have significant differences in fitness levels, which in turn, have significant implication in performance. In general, ballet dancers have been consistently found to demonstrate reduced fitness levels than other athletic populations $[8,14,41]$. It has been postulated that contemporary dancers may also be relatively unfit as the main sections of their training (i.e. class and rehearsal) do not adequately stress the physiological system [54-56].

Contemporary dance emerged at the beginning of the 20th century as a breakaway from the rigid constraints of classical ballet which itself started as a performance art in the French courts in the 16th and 17th century. Despite its many years of life, contemporary dance has not yet developed the fitness standards which might be considered as appropriate for the task [26]. Furthermore, it remains unclear whether improved physical fitness affects aspects of dance performance in student and professional contemporary dancers. Therefore, the aims of this systematic review were to investigate: a) the aerobic/anaerobic fitness, muscular strength and body composition characteristics of contemporary dancers and b) whether supplementary exercise training improves aspects of contemporary dance performance.

\section{Method}

$\nabla$

Three databases [Medline, Cochrane and the Cumulative Index to Nursing \& Allied Health (CINAHL) research database] were searched to identify publications in English (published from 1978 until May 2008) regarding fitness components of contemporary dancers. The Medical Subject Heading (MeSH) terms "physical fitness", "exercise", "performance", "training", "aerobic", "anaerobic", "strength", "body composition", "fat free mass", were employed in combination with "modern dance", "contemporary dance", "dance" and "dancers". Full articles were retrieved for assessment if the information in the abstract fulfilled the following inclusion criteria: (i) studying any of the main fitness components in combination with contemporary dance, and (ii) involving professional dancers and dance students in vocational

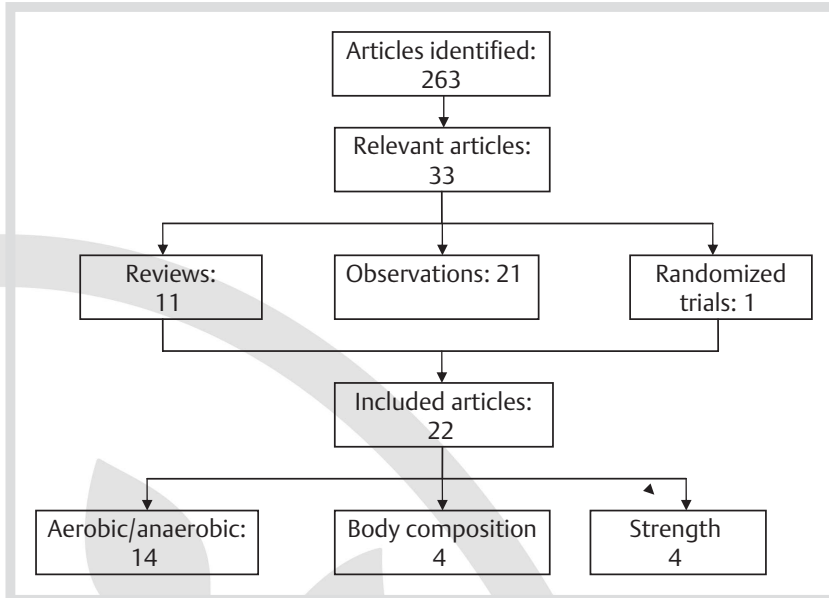

Fig. 1 Studies included in the systematic review.

and university training. We chose to include dance students enrolled in both vocational and university courses because their training involves contemporary dance alone or in combination with other dance styles. Editorials, conference proceedings and studies incorporating only ballet or other dance styles alone were excluded. If the abstract did not provide sufficient information for this process, then the full-text manuscript was examined. A flow diagram of the studies identified and included appears in $\odot$ Fig. 1.

\section{Results}

$\nabla$

Initial search revealed 263 articles. From those articles, 24 fulfilled the inclusion criteria and thus were included for further analysis. The references of all of these articles were examined in order to further identify relevant publications; nine more studies were found. From the 33 included articles, 11 publications were reviews (none of which was a systematic review); only one article was a randomised controlled trial (RCT), while the remaining 21 publications were non-randomised research investigations. The RCT investigated the effects of a combined aerobic and strength exercise intervention on contemporary dance performance. From the non-randomised studies, 13 primarily investigated aerobic/anaerobic related physiological variables in relation to contemporary dancers, four studies were on strengthrelated parameters and four were on body composition. The comparisons for maximal oxygen uptake $\left(\mathrm{VO}_{2 \max }\right)$ and body composition characteristics between female professional and student contemporary dancers as well as the equivalent values from other sports appear in 8 Table 1.

\section{Aerobic|Anaerobic fitness}

A number of authors have investigated the aerobic and anaerobic fitness levels of contemporary dancers and dance students, using laboratory-based maximal exertion tests. From the total of the 13 studies, $V_{2 \max }$ was found to range from $39.2 \pm 1.9$ to $50.7 \pm 7.5 \mathrm{ml} \cdot \mathrm{kg}^{-1} \cdot \mathrm{min}^{-1}$. However, these data refer to both male and female professional dancers and dance students. As such, different values for males and females could not be reported in - Table 1.

Three authors investigated the $\mathrm{VO}_{2 \max }$ among different level of contemporary dance students and they found no significant dif- 
Table 1 Maximal oxygen uptake and body composition characteristics of female contemporary professional dancers and dance students compared to other athletes.

\begin{tabular}{|c|c|c|c|c|c|}
\hline \multirow[t]{2}{*}{ Sport/Activity } & \multirow[t]{2}{*}{ Level } & \multirow{2}{*}{$\begin{array}{l}\mathrm{VO}_{2 \max } \\
\left(\mathrm{ml} . \mathrm{kg}^{1} \mathrm{~min}^{-1}\right)\end{array}$} & \multicolumn{2}{|c|}{ Body Composition) } & \multirow[t]{2}{*}{ References } \\
\hline & & & Fat Mass (\% & Fat-Free Mass (kg) & \\
\hline contemporary & professional & 49.1 & 21.4 & 42.9 & {$[9,13,17]$} \\
\hline dance & students & 39.2 & 21.3 & 42.5 & {$[50,59]$} \\
\hline \multirow[t]{2}{*}{ ballet } & professional & 42.2 & 17.4 & 42.3 & {$[13,21,46]$} \\
\hline & students & 40.8 & 19.9 & 41.5 & [50] \\
\hline gymnastics & & 49.6 & 14.4 & 33.7 & {$[16,48]$} \\
\hline football & & 50.0 & 20 & 50.3 & {$[7,35]$} \\
\hline endurance running & & 77 & 35.8 & 23.8 & {$[10,34]$} \\
\hline volleyball & & 46.5 & 53.2 & 23.4 & {$[32,45]$} \\
\hline swimming & & 58 & 47.6 & 20.2 & {$[3,42]$} \\
\hline sedentary & & 44 & 24.4 & 26.5 & {$[4,36,46]$} \\
\hline
\end{tabular}

ferences existed in a) university, graduate and professional [55], b) performing and recreational adolescent dancers [38] and c) intermediate and advanced dance students [12]. One study revealed that $\mathrm{VO}_{2 \max }$ was higher in students undertaking ballet and modern dance as majors, compared to other dance combinations in a vocational institution [15]. Moreover, ballet and contemporary dance students did not demonstrate significant differences in cardiorespiratory fitness [50], while professional contemporary dancers exhibited significant higher values of $\mathrm{VO}_{2 \max }$ than their ballet counterparts [13].

In comparison to non-dancers, it was found that the $\mathrm{VO}_{2 \max }$ values of intermediate and advanced dancers' was significantly increased. However, no significant differences were detected between beginners and non-dancers [12]. These results are in line with the study of Novak [36] who found that female dancers had a significantly higher mean $\mathrm{VO}_{2 \max }$ value compared to sedentary females of a similar age range. Despite the consistently higher aerobic capacity of dancers compared to controls, an investigation revealed no significant differences for all structural and functional cardiac indices between full-time contemporary dance students and age-gender matched controls [51].

Anaerobic power, determined by the Wingate test, was primarily investigated in two studies. Chatfield and colleagues [12] reported that advanced level contemporary dancers showed anaerobic capacity mean values of $907.5 \pm 140.7 \mathrm{Kgm} .30 \mathrm{sec}^{-1}$ which was somewhat lower (but not significantly) compared to beginners $\left(922.5 \pm 195.4 \mathrm{Kgm} .30 \mathrm{sec}^{-1}\right)$. These results, however, were relatively higher (but not significantly) compared to nondancers ( Table 2). Non significant differences were also detected in anaerobic mean power between adolescent dance students compared to either recreational dancers or non-dancers [38]. Significant differences were instead depicted in post exercise blood lactate levels, where professional contemporary dancers exhibited higher values than their ballet counterparts [13].

By investigating the effects of supplementary exercise training on jumping ability and dance performance $[11,25]$, these recent studies form the only known examples where aspects of fitness and contemporary dance performance were simultaneously considered. Brown and colleagues [11] recruited three experienced dance teachers to assess four aspects of the dancers jumping ability including: the aptitude to hang suspended in the air during a jump (ballon), height of the jumps, the ability to point the feet in the air and the overall aesthetic jumping ability. Overall aesthetic competence and dance technique were assessed by
Koutedakis and colleagues [25], who specially designed a dance performance test. By adopting the marking procedures used in sports (e.g. gymnastics and ice skating), two teachers and former professional dancers, were recruited as markers. Combined evidence from both studies reveals that aerobic and strength training increases $\mathrm{VO}_{2 \max }$, strength and aspects of dance performance in contemporary dance students $[11,25]$. Most importantly, these results are in line with the only RCT which is currently available in dance literature [25].

- Table 2 depicts the individual results from all studies conducted in relation to aerobic/anaerobic capacity of contemporary dancers.

\section{Muscular strength}

One maximum repetition for leg press in dance students has been found to range from $183.3 \pm 30.9$ to $222.7 \pm 65.0 \mathrm{~kg}$, while knee curl and extension ranges from $34.8 \pm 4.5$ to $40.0 \pm 5.7 \mathrm{~kg}$ and from $58.7 \pm 6.5$ to $62.5 \pm 9.1 \mathrm{~kg}$, respectively [11]. Moreover, muscular strength and power of knee and ankle in both intermediate and/or advanced dance students has been found to be not significantly different between them or when compared to sedentary individuals [12]. No significant differences were observed in the quadriceps and hamstring peak torque between ballet and contemporary dance students and professionals [13]; however, in comparison to ballet, contemporary dancers reported higher scores in muscular endurance but lower compared to folk dancers [44]. Finally, it was found [27] that the knee extensor and flexor muscle peak power of female professional contemporary dancers was $151.0 \pm 26.0$ and $63.0 \pm 11.0 \mathrm{Nm}$ at $1.04 \mathrm{rad} / \mathrm{sec}$ while at $4.19 \mathrm{rad} / \mathrm{sec}$ the equivalent values were $83.0 \pm 11.0$ and $60.0 \pm 8.4 \mathrm{Nm}$. Isokinetic measurements of muscular strength [17] revealed that semi-professional dancers compared to athletes, have a greater quads muscle output during a five sec maximal voluntary isometric contraction but they do not jump higher than controls.

Two studies investigated the effects of supplementary strength training (both plyometric and weight training) on dance students, and both studies revealed that a significant increase in muscular strength resulted in significant benefits for enhancing aesthetic jump performance [11] and overall aesthetic competence and dance technique, assessed via a specifically designed dance performance test [25].

- Table 3 depicts the individual evidence from all studies conducted in relation to strength related parameters of contemporary dancers. 
Table 2 Studies primarily investigating the aerobic/anaerobic capacity of contemporary dancers.

\begin{tabular}{|c|c|c|c|}
\hline Author (reference) & Participants & Method & Results \\
\hline \multirow[t]{4}{*}{ Koutedakis et al. 2007 [25] } & 32 dance students & $\begin{array}{l}\mathrm{F}=2-3 \mathrm{~h} / \mathrm{wk} \\
\text { Intensity-Aerobic }= \\
70-75 \% \text { of } \mathrm{VO}_{2} \max \end{array}$ & $\begin{array}{l}\text { Intervention group before intervention: } \\
\text { VO }_{2 \text { max }}: 50.7 \pm 7.5 \mathrm{ml} \cdot \mathrm{kg}^{-1} \cdot \mathrm{min}^{-1} \\
\text { Strength both legs: } 90.6 \pm 16.0 \mathrm{~kg} \\
\text { Skinfolds: } 39.4 \pm 10.5 \mathrm{~mm}\end{array}$ \\
\hline & $\begin{array}{l}\text { intervention group } \\
\mathrm{n}=19\end{array}$ & $\begin{array}{l}\text { Intensity-Strength = } 6 \text { weeks: } \\
<70 \% \text { of } 1 \\
\text { repetition maximum } \\
\text { high repetitions }\end{array}$ & $\begin{array}{l}\text { Intervention group after intervention: } \\
\text { VO }_{2 \mathrm{max}}: 56.6 \pm 9.3 \mathrm{ml} \cdot \mathrm{kg}^{-1} \cdot \mathrm{min}^{-1 *} \\
\text { Strength both legs: } 102.0 \pm 17.4 \mathrm{~kg} * \\
\text { Skinfolds: } 35.7 \pm 9.3 \mathrm{~mm}\end{array}$ \\
\hline & control group $n=13$ & $\begin{array}{l}6 \text { weeks: }>70 \% \text { of } 1 \text { repeti- } \\
\text { tion maximum low repetitions }\end{array}$ & $\begin{array}{l}\text { Control group before intervention: } \\
\mathrm{VO}_{2 \mathrm{max}}: 49.2 \pm 5.5 \mathrm{ml} \cdot \mathrm{kg}^{-1} \cdot \mathrm{min}^{-1} \\
\text { Strength both legs: } 94.1 \pm 15.8 \mathrm{~kg} \\
\text { Skinfolds: } 40.9 \pm 11.7 \mathrm{~mm}\end{array}$ \\
\hline & RCT & $\mathrm{PD}=12$ weeks & 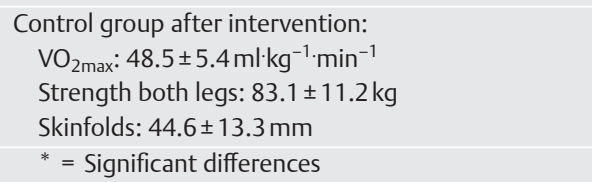 \\
\hline \multirow[t]{3}{*}{ White et al. 2004 [50] } & $\begin{array}{l}\text { ballet students } \\
\mathrm{n}=10\end{array}$ & $\begin{array}{l}\text { Aerobic capacity and body } \\
\text { composition }\end{array}$ & $\begin{array}{l}\text { Ballet: } \\
\text { VO }{ }_{2 \max }: 40.8 \pm 1.6 \mathrm{ml} \cdot \mathrm{kg}^{-1} \cdot \mathrm{min}^{-1} \\
\text { \%BF: } 19.9 \pm 1.5 \\
\text { FFM: } 41.5 \pm 1.1 \mathrm{~kg}\end{array}$ \\
\hline & $\begin{array}{l}\text { contemporary } \\
\text { students } \mathrm{n}=7\end{array}$ & & $\begin{array}{l}\text { Contemporary: } \\
\text { VO }_{2 \max }: 39.2 \pm 1.9 \mathrm{ml} \cdot \mathrm{kg}^{-1} \cdot \mathrm{min}^{-1} \\
\% \mathrm{BF}: 19.3 \pm 1.4 \\
\text { FFM: } 43.2 \pm 1.6 \mathrm{~kg}\end{array}$ \\
\hline & & & No significant differences \\
\hline \multirow[t]{4}{*}{ Wyon et al. 2002 [55] } & $\begin{array}{l}27 \text { dancers } \\
\text { university } n=10\end{array}$ & $\begin{array}{l}\text { Cardiorespiratory responses } \\
\left(\text { mean } \mathrm{VO}_{2}\right)\end{array}$ & $\begin{array}{l}\text { University: } \\
\text { Mean } \mathrm{VO}_{2}: 16.8 \pm 2.3 \mathrm{ml} \cdot \mathrm{kg}^{-1} \cdot \mathrm{min}^{-1}\end{array}$ \\
\hline & graduate $n=7$ & to dance class & $\begin{array}{l}\text { Graduate: } \\
\quad \text { Mean } \mathrm{VO}_{2}: 20.4 \pm 4.8 \mathrm{ml} \cdot \mathrm{kg}^{-1} \cdot \mathrm{min}^{-1}\end{array}$ \\
\hline & professional $\mathrm{n}=10$ & & $\begin{array}{l}\text { Professional: } \\
\quad \text { Mean } \mathrm{VO}_{2}: 18.3 \pm 3.8 \mathrm{ml} \cdot \mathrm{kg}^{-1} \cdot \mathrm{min}^{-1}\end{array}$ \\
\hline & & & No significant differences \\
\hline \multirow[t]{2}{*}{ Whyte et al. 2003 [51] } & $\begin{array}{l}\text { dance students } \\
n=44 \\
\text { females non-active } \\
n=30\end{array}$ & $\begin{array}{l}\text { Echocardiography and } \\
\text { Electrocardiography }\end{array}$ & $\begin{array}{l}\text { No significant differences between dancers and } \\
\text { controls for structural and functional cardiac indices. } \\
\text { Contemporary students: } \\
\text { \% BF: } 18.7 \pm 3.4 \\
\text { FFM: } 48.2 \pm 6.3 \mathrm{~kg}\end{array}$ \\
\hline & & & $\begin{array}{l}\text { Controls: } \\
\text { \%BF: } 19.9 \pm 4.4 \\
\text { FFM: } 47.3 \pm 5.8 \mathrm{~kg}\end{array}$ \\
\hline Redding et al. 2003 [40] & $\begin{array}{l}19 \text { professional } \\
\text { females } n=12 \\
\text { males } n=7\end{array}$ & $\begin{array}{l}\text { Validity of using } \mathrm{HR} \text { as a } \\
\text { predictor of mean } \mathrm{VO}_{2} \\
\text { consumption in dance }\end{array}$ & $\begin{array}{l}\text { It is unacceptable to predict the mean } \mathrm{VO}_{2} \text { from } \mathrm{HR} \\
\text { values, based on the } \mathrm{HR}-\mathrm{VO}_{2} \text { relationship established } \\
\text { from a progressive treadmill protocol }\end{array}$ \\
\hline \multirow[t]{2}{*}{ Wyon et. al 2003 [57] } & $\begin{array}{l}56 \text { dancers } \\
\text { females } n=32\end{array}$ & $\begin{array}{l}\mathrm{VO}_{2 \max } \text { during a dance } \\
\text { specific aerobic test }\end{array}$ & $\begin{array}{l}\text { Females: } \\
\qquad \mathrm{VO}_{2 \max }: 46.0 \pm 3.5 \mathrm{ml} \cdot \mathrm{kg}^{-1} \cdot \mathrm{min}^{-1}\end{array}$ \\
\hline & males $n=24$ & & $\begin{array}{l}\text { Males: } \\
\qquad \mathrm{VO}_{2 \max }: 56.0 \pm 3.5 \mathrm{ml} \cdot \mathrm{kg}^{-1} \cdot \mathrm{min}^{-1}\end{array}$ \\
\hline \multirow[t]{3}{*}{ Padfield et al. 1993 [38] } & $\begin{array}{l}\text { performing adolescent } \\
\text { dancers } n=24\end{array}$ & $\begin{array}{l}\mathrm{VO}_{2 \max } \text { \% } \% \mathrm{BF}, \text { jump } \\
\text { height, anaerobic mean } \\
\text { power }\end{array}$ & $\begin{array}{l}\text { Performing dancers: } \\
\mathrm{VO}_{2 \max }: 45.6 \pm 4.8 \mathrm{ml} \cdot \mathrm{kg}^{-1} \cdot \mathrm{min}^{-1} \\
\text { \%BF: } 8.7 \pm 6.5 \\
\text { Jump height: } 26.7 \pm 3.5 \mathrm{~cm} \\
\text { Anaerobic mean power: } 6.6 \pm 0.7 \text { Watts } \mathrm{kg}^{-1}\end{array}$ \\
\hline & $\begin{array}{l}\text { recreational adoles } \\
\text { cent dancers } n=16\end{array}$ & & $\begin{array}{l}\text { Recreational dancers: } \\
\mathrm{VO}_{2 \max }: 46.3 \pm 6.0 \mathrm{ml} \cdot \mathrm{kg}^{-1} \cdot \mathrm{min}^{-1} \\
\% \mathrm{BF}: 9.7 \pm 7.6 \\
\text { Jump height: } 25.1 \pm 6.6 \mathrm{~cm} \\
\text { Anaerobic mean power: } 6.2 \pm 0.9 \text { Watts } \mathrm{kg}^{-1}\end{array}$ \\
\hline & & & No significant differences \\
\hline Wyon et al. 2004 [54] & $\begin{array}{l}\text { dance students (males } \\
\text { and females) } n=40\end{array}$ & $\begin{array}{l}\text { Mean } \mathrm{VO}_{2} \text { during class, } \\
\text { performance and rehearsal }\end{array}$ & $\begin{array}{l}\text { Females: } \\
\text { Class mean } \mathrm{VO}_{2}: 17.4 \pm 2.7 \mathrm{ml} \cdot \mathrm{kg}^{-1} \cdot \mathrm{min}^{-1} \\
\text { Rehearsal mean } \mathrm{VO}_{2}: 10.2 \pm 6.6 \mathrm{ml} \cdot \mathrm{kg}^{-1} \cdot \mathrm{min}^{-1} \\
\text { Performance mean } \mathrm{VO}_{2}: 23.3 \pm 3.8 \mathrm{ml} / \mathrm{kg}^{-1} \cdot \mathrm{min}^{-1}\end{array}$ \\
\hline
\end{tabular}


Table 2 Continued.

\begin{tabular}{|c|c|c|c|}
\hline & & & $\begin{array}{l}\text { Males: } \\
\text { Class mean } \mathrm{VO}_{2}: 22.1 \pm 5.9 \mathrm{ml} \cdot \mathrm{kg}^{-1} \cdot \mathrm{min}^{-1} \\
\text { Rehearsal mean } \mathrm{VO}_{2}: 17.2 \pm 3.3 \mathrm{ml} \cdot \mathrm{kg}^{-1} \cdot \mathrm{min}^{-1} \\
\text { Performance mean } \mathrm{VO}_{2}: 24.9 \pm 5.8 \mathrm{ml} \cdot \mathrm{kg}^{-1} \cdot \mathrm{min}^{-1}\end{array}$ \\
\hline & & & $\begin{array}{l}\text { Performance had a significantly greater } \\
\text { physiological demand than rehearsal and class. }\end{array}$ \\
\hline \multirow[t]{6}{*}{ Wyon et al. 2005 [56] } & $\begin{array}{l}\text { professional dancers } \\
n=17 \\
\text { company } 1 n=10\end{array}$ & $\begin{array}{l}\text { Company } 1 \\
\text { Aerobic supplemental training } \\
\text { no guidance provision and }\end{array}$ & $\begin{array}{l}\text { Pre assessment company } 1 \\
\text { Mean HR: } 166 \pm 10.65 \mathrm{~b} \cdot \mathrm{min}^{-1} \\
\mathrm{BL}: 2.1 \pm 0.9 \mathrm{Mmol} \cdot \mathrm{L}^{-1}\end{array}$ \\
\hline & & $\begin{array}{l}\text { time } \\
\mathrm{PD}=8 \text { weeks }\end{array}$ & $\begin{array}{l}\text { Post assessment Company } 1 \text { : } \\
\text { Mean HR: } 155 \pm 12.9 \mathrm{~b} \cdot \mathrm{min}^{-1} \\
\text { BL: } 1.5 \pm 0.8 \mathrm{Mmol} \cdot \mathrm{L}^{-1}\end{array}$ \\
\hline & & & Significant differences \\
\hline & company $2 \mathrm{n}=7$ & $\begin{array}{l}\text { Company } 2 \\
\text { Dance training only }\end{array}$ & $\begin{array}{l}\text { Pre assessment company } 2 \text { : } \\
\text { Mean HR: } 189 \pm 4.19 \mathrm{~b} \cdot \mathrm{min}^{-1} \\
\text { BL: } 3.4 \pm 1.1 \mathrm{Mmol} \cdot \mathrm{L}^{-1}\end{array}$ \\
\hline & & & $\begin{array}{l}\text { Post assessment company } 2 \text { : } \\
\text { Mean HR: } 179 \pm 4.8 \mathrm{~b} \cdot \mathrm{min}^{-1} \\
\text { BL: } 2.8 \pm 1.1 \mathrm{Mmol} \cdot \mathrm{L}^{-1}\end{array}$ \\
\hline & intervention non RCT & & No significant differences \\
\hline \multirow[t]{3}{*}{ Novak et al. 1978 [36] } & $\begin{array}{l}\text { female dance students } \\
\mathrm{n}=12\end{array}$ & $\mathrm{VO}_{2 \max }$ and $\% \mathrm{BF}$ & $\begin{array}{l}\text { Dance students: } \\
V_{2 \max }: 41.5 \pm 6.7 \mathrm{ml} \mathrm{kg}^{-1} \cdot \mathrm{min}^{-1} \\
\% \mathrm{BF}: 20.5 \pm 4.6\end{array}$ \\
\hline & $\begin{array}{l}\text { sedentary females } \\
\mathrm{n}=12\end{array}$ & & $\begin{array}{l}\text { Controls: } \\
\qquad \mathrm{VO}_{2 \mathrm{max}}: 36.8 \pm 5.5 \mathrm{ml} \cdot \mathrm{kg}^{-1} \cdot \mathrm{min}^{-1} \\
\text { \%BF: } 26.5 \pm 3.6\end{array}$ \\
\hline & & & Significant differences in $\mathrm{VO}_{2 \max }$ and \% $\mathrm{BF}$ \\
\hline \multirow[t]{11}{*}{ Chatfield et al. 1990 [12] } & & $\mathrm{VO}_{2 \max } \% \mathrm{BF}$, knee and & $\mathrm{VO}_{2 \max }$ \\
\hline & non dancers $n=8$ & ankle strength, knee and & Non dancers: $\quad 36.4 \pm 4.8 \mathrm{ml} \cdot \mathrm{kg}^{-1} \cdot \mathrm{min}^{-1}$ \\
\hline & $\begin{array}{l}\text { beginner students } \\
\mathrm{n}=14\end{array}$ & ankle power, WAT power & Beginners: $\quad 40.4 \pm 4.9 \mathrm{ml} \cdot \mathrm{kg}^{-1} \cdot \mathrm{min}^{-1}$ \\
\hline & $\begin{array}{l}\text { intermediate students } \\
\mathrm{n}=11\end{array}$ & & Intermediate: $42.5 \pm 4.3 \mathrm{ml} \cdot \mathrm{kg}^{-1} \cdot \mathrm{min}^{-1}$ \\
\hline & $\begin{array}{l}\text { professional dancers } \\
\mathrm{n}=8\end{array}$ & & Professional: $43.6 \pm 2.3 \mathrm{ml} \cdot \mathrm{kg}^{-1} \cdot \mathrm{min}^{-1}$ \\
\hline & & & $\begin{array}{l}\text { Significant differences between non-dancers and } \\
\text { intermediate and non- dancers and professionals }\end{array}$ \\
\hline & & & $\begin{array}{l}\text { \%BF: } \\
\text { Non dancers: } 27.8 \pm 4.4 \\
\text { Beginners: } 23.7 \pm 4.8 \\
\text { Intermediate: } 20.9 \pm 4.6 \\
\text { Professional: } 18.1 \pm 2.3\end{array}$ \\
\hline & & & $\begin{array}{l}\text { Significant differences between non-dancers and } \\
\text { intermediate, non-dancers and professionals, and } \\
\text { professional and beginners }\end{array}$ \\
\hline & & & $\begin{array}{l}\text { No significant differences in knee and ankle } \\
\text { strength and power between all groups }\end{array}$ \\
\hline & & & $\begin{array}{l}\text { WAT capacity: } \\
\text { Non Dancers: } 828.38 \pm 161.2 \mathrm{kgm} \cdot 30 \mathrm{sec}^{-1} \\
\text { Beginners: } 922.50 \pm 195.4 \mathrm{kgm} \cdot 30 \mathrm{sec}^{-1} \\
\text { Intermediate: } 917.73 \pm 120.1 \mathrm{kgm} \cdot 30 \mathrm{sec}^{-1} \\
\text { Professional: } 907.50 \pm 140.7 \mathrm{kgm} \cdot 30 \mathrm{sec}^{-1}\end{array}$ \\
\hline & & & No significant differences \\
\hline \multirow[t]{3}{*}{ Dahlstrom et al. 1996 [15] } & $\begin{array}{l}\text { dance students } \\
\text { ballet + contemporary } \\
\mathrm{n}=10\end{array}$ & $\mathrm{VO}_{2 \max }$ & $\begin{array}{l}\text { Ballet }+ \text { contemporary } \mathrm{VO}_{2 \max } \text { : } \\
51.2 \pm 11.4 \mathrm{ml} \cdot \mathrm{kg}^{-1} \cdot \mathrm{min}^{-1}\end{array}$ \\
\hline & $\begin{array}{l}\text { contemporary }+ \text { jazz } \\
\mathrm{n}=30\end{array}$ & & Contemporary + jazz $\mathrm{VO}_{2 \max }: \quad 45.8 \pm 8.7 \mathrm{ml} \cdot \mathrm{kg}^{-1} \cdot \mathrm{min}^{-1}$ \\
\hline & $\begin{array}{l}\text { contemporary }+ \\
\text { character } \mathrm{n}^{+}=\end{array}$ & & Contemporary + character $\mathrm{VO}_{2 \max }: \quad 46.6 \pm 12.2 \mathrm{ml} \cdot \mathrm{kg}^{-1} \cdot \mathrm{min}^{-1}$ \\
\hline Chmelar et al. 1988 [13] & $\begin{array}{l}\text { professional } \\
\text { contemporary } n=9\end{array}$ & $\begin{array}{l}\mathrm{VO}_{2 \max }, \mathrm{BL}, \% \mathrm{BF} \\
\mathrm{QPT} / \mathrm{BW}, \mathrm{HPT} / \mathrm{BW}\end{array}$ & $\begin{array}{l}\text { Contemporary professional: } \\
\text { VO }_{2 \text { max }}: 49.1 \pm 5.9 \mathrm{ml}^{-1} \mathrm{~kg}^{-1} \cdot \mathrm{min}^{-1} \\
\text { BL: } 9.7 \pm 1.4 \mathrm{mM} \cdot \mathrm{L}^{-1} \\
\% \text { BF: } 12.2 \pm 2.1 \\
60^{\circ} / \mathrm{sec} \text { QPT/BW: } 75.7 \pm 13.1 \%\end{array}$ \\
\hline
\end{tabular}


Table 2 Continued.

professional ballet $n=9$

contemporary

students $n=11$

ballet students $n=10$
$60 \%$ sec HPT/BW: $43.2 \pm 5.9 \%$

$180^{\circ} / \mathrm{sec}$ QPT/BW: $47.9 \pm 6.1 \%$

$180^{\circ} / \mathrm{sec} \mathrm{HPT} / \mathrm{BW}: 36.4 \pm 4.1 \%$

Professional ballet:

$\mathrm{VO}_{2 \max }: 42.2 \pm 2.9 \mathrm{ml} \cdot \mathrm{kg}^{-1} \cdot \mathrm{min}^{-1}$ *

BL: $6.0 \pm 1.5 \mathrm{mM} \cdot \mathrm{L}^{-1}$ *

\%BF: $14.1 \pm 1.9$

$60^{\circ} / \mathrm{sec}$ QPT/BW: $73.7 \pm 12.4 \%$

$60^{\circ} / \mathrm{sec}$ HPT/BW: $50.5 \pm 6.7 \%$

$180^{\circ} / \mathrm{sec}$ QPT/BW: $46.5 \pm 11.4 \%$

$180^{\circ} / \mathrm{sec}$ HPT/BW: $42.3 \pm 9.9 \%$

Contemporary students:

$\mathrm{VO}_{2 \max }: 47.5 \pm 3.1 \mathrm{ml} \cdot \mathrm{kg}^{-1} \cdot \mathrm{min}^{-1}$

BL: $9.0 \pm 2.4 \mathrm{mM} \cdot \mathrm{L}^{-1}$

\%BF: $14.7 . \pm 3.4$

$60^{\circ} / \mathrm{sec}$ QPT/BW: $78.8 \pm 14.0 \%$

$60 \% \mathrm{sec} \mathrm{HPT/BW:} 45.1 \pm 6.6 \%$

$180^{\circ} / \mathrm{sec}$ QPT/BW: $50.9 \pm 9.1 \%$

$180^{\circ} / \mathrm{sec} \mathrm{HPT} / \mathrm{BW}: 37.4 \pm 5.1 \%$

Ballet students:

$\mathrm{VO}_{2 \max }: 47.0 \pm 2.1 \mathrm{ml} \cdot \mathrm{kg}^{-1} \cdot \mathrm{min}^{-1}$

BL: $9.5 \pm 0.9 \mathrm{mM} \cdot \mathrm{L}^{-1}$

\%BF: 14.2. \pm 3.2

$60^{\circ} / \mathrm{sec}$ QPT/BW: $74.7 \pm 10.1 \%$

$60 \%$ sec HPT/BW: $46.2 \pm 5.5 \%$

$180^{\circ} / \mathrm{sec}$ QPT/BW: $45.5 \pm 6.3 \%$

$180^{\circ} / \mathrm{sec} \mathrm{HPT} / \mathrm{BW}: 35.5 \pm 5.0 \%$

* = significant difference between ballet and contemporary

professionals and dance students (ballet and contemporary)

$\mathrm{VO}_{2 \text { max }}: 45.0 \pm 3.9 \mathrm{ml} \cdot \mathrm{kg}^{-1} \cdot \mathrm{min}^{-1}$

O’Mailia et al. $2002[37] \quad$ female dancers $n=14 \quad \mathrm{VO}_{2 \max }$

max $=$ maximal oxygen uptake; mean $\mathrm{VO}_{2}=$ mean volume of oxygen uptake; \% $\mathrm{BF}=$ percentage

body fat; $F F M=$ fat-free mass; $H R=$ heart rate

$\mathrm{VO}_{2 \max }=$ maximal oxygen uptake; \% $\mathrm{BF}=$ percentage body fat; mean $\mathrm{VO}_{2}=$ mean volume of oxygen uptake; $\mathrm{RCT}$ : randomized control trial; $\mathrm{PD}=$ programme duration; $\mathrm{HR}=$ heart rate; $\mathrm{BL}=$ blood lactate; $\mathrm{WAT}=$ Wingate anaerobic power test; $\mathrm{QPT} / \mathrm{BW}=$ quadriceps peak torque/body weight; $\mathrm{HPT} / \mathrm{BW}=$ hamstring peak torque/body weight

\section{Body composition}

An investigation into body composition of female dancers revealed that, using the skinfolds method, percentage body fat (\%BF) was found to range from 13.0 to $26.9 \%$. In the same sample of participants, using dual energy X ray absorptiometry (DXA), values for \%BF ranged from 10.3 to $30.4 \%$. The fat-free mass (FFM), as determined by DXA, was $42.6 \pm 3.3 \mathrm{~kg}$ (range 35.6-50.1) [59]. Regarding different level of dancers, it has been found that performing and recreational adolescent dance students do not significantly differ in \%BF [38]. In all these aforementioned studies, the total number of dance students derived from both ballet and modern dance training. In other studies, however, which indeed separated the dance students into ballet and contemporary, no significant differences were found either in \%BF or FFM between these two dance styles $[13,50]$. Significant lower \%BF values were instead found in professional contemporary dancers compared to beginner dance students [12].

Female dance students and graduates were found to have a significantly lower \%BF than age-matched non-active females [36]. In addition, significant differences were also found between advanced dancers and non-dancers, as well as intermediate dance students and non-dancers [12].

Results from one RCT and one non randomized intervention revealed that supplementary aerobic and/or strength training does not elicit significant changes in \%BF of dance students $[11,25]$. O Table 4 depicts the evidence from all studies primarily investigating body composition characteristics of contemporary dancers.

\section{Discussion}

$\nabla$

The aim of this systematic review was to investigate a) the aerobic/ anaerobic fitness, strength levels and body composition characteristics of contemporary dancers and b) if supplementary training is effective in improving aspects of dance performance. To our knowledge, this is the first review systematically investigating all these fitness components in relation to contemporary dance. Unlike most ballet dancers, contemporary dancers may have a multidisciplinary background, which includes other sport activities such as gymnastics [26]. This is also the case in other sports such as football and water polo, where the players used to be runners and swimmers, respectively. This sample heterogeneity as well as the different training demands between contemporary and ballet dance, may result in different levels of fitness and strength. Moreover, it is important to highlight that contemporary choreographers require different artistic, technical and physical demands during auditions [52]. Hence, it seems reasonable to suggest that contemporary dancers with sporting background might be also advantaged during auditions. Other differences between ballet and contemporary dancers have been reported to be injury sites and rates as well as the biomechanical mechanisms that cause these injuries [29]. Moreover, results from separate studies investigating physiological demands of contemporary [54] and ballet [14] class and performance, suggest that the two dance styles may also differ in their cardiorespiratory demands. For these reasons it was decided not to report research evidence for dancers as a whole, but to focus on the contemporary style, and differenti- 
Table 3 Studies primarily investigating muscle strength levels of contemporary dancers.

\begin{tabular}{|c|c|c|c|}
\hline $\begin{array}{l}\text { Author } \\
\text { (reference) }\end{array}$ & Participants & Method & Results \\
\hline \multirow[t]{11}{*}{$\begin{array}{l}\text { Koutedakis } \\
\text { et al.1997 [27] }\end{array}$} & $\begin{array}{l}\text { male ballet and contemporary } \\
\text { professionals } n=20\end{array}$ & $\begin{array}{l}\text { Knee extensors and flexor } \\
\text { muscle peak torques, \%BF, } \\
\text { FFM }\end{array}$ & $\begin{array}{l}\text { Males: Knee flexion at } 1.04(\mathrm{rad} / \mathrm{sec}): 121 \pm 15 \mathrm{Nm} \\
\text { Knee extension at } 1.04(\mathrm{rad} / \mathrm{sec}): 248 \pm 24 \mathrm{Nm} \\
\text { Sum: } 5.8 \pm 0.5 \mathrm{Nm} / \mathrm{kg} \mathrm{FFM}\end{array}$ \\
\hline & & & $\begin{array}{l}\text { Knee flexion at } 4.19(\mathrm{rad} / \mathrm{sec}): 81 \pm 10 \mathrm{Nm} \\
\text { Knee extension at } 4.19(\mathrm{rad} / \mathrm{sec}): 131 \pm 12 \mathrm{Nm} \\
\text { Sum: } 3.3 \pm 3.1 \mathrm{Nm} / \mathrm{kg} \text { FFM }\end{array}$ \\
\hline & $\begin{array}{l}\text { female ballet and contemporary } \\
\text { professionals } n=22\end{array}$ & & $\begin{array}{l}\text { Females: Knee flexion at } 1.04 \text { (rad/sec): } 63 \pm 11 \mathrm{Nm} \\
\text { Knee extension at } 1.04 \text { (rad/sec): } 151 \pm 26 \mathrm{Nm} \\
\text { Sum: } 4.6 \pm 0.4 \mathrm{Nm} / \mathrm{kg} \text { FFM }\end{array}$ \\
\hline & & & $\begin{array}{l}\text { Knee flexion at } 4.19(\mathrm{rad} / \mathrm{sec}): 60 \pm 8.4 \mathrm{Nm} \\
\text { Knee extension at } 4.19(\mathrm{rad} / \mathrm{sec}): 83 \pm 11 \mathrm{Nm} \\
\text { Sum: } 3.1 \pm 0.2 \mathrm{Nm} / \mathrm{kg} \mathrm{FFM}\end{array}$ \\
\hline & & & $\begin{array}{l}\text { Significant differences between males and females in knee flex- } \\
\text { ion, extension and sum at } 1.04(\mathrm{rad} / \mathrm{sec}) \text {, and in knee flexion and } \\
\text { extension at } 4.19(\mathrm{rad} / \mathrm{sec})\end{array}$ \\
\hline & & & Males: \%BF: $14.1 \pm 2.4$ \\
\hline & & & Females: \%BF: $19.2 \pm 5.8$ \\
\hline & & & Significant difference \\
\hline & & & Males: FFM: $63.0 \pm 5.1 \mathrm{~kg}$ \\
\hline & & & Females: FFM: $45.3 \pm 4.1 \mathrm{~kg}$ \\
\hline & & & Significant difference \\
\hline \multirow[t]{13}{*}{$\begin{array}{l}\text { Harley et al. } \\
2002[17]\end{array}$} & $\begin{array}{l}\text { female semi professional dancers } \\
\mathrm{n}=11 \text { (semi professional) }\end{array}$ & $\begin{array}{l}\text { Quads strength, jump } \\
\text { height, \%BF, FFM }\end{array}$ & $\begin{array}{l}\text { Dancers had significantly greater peak and mean force output in } \\
\text { the } 5 \text { sec maximal voluntary isometric leg extension tests ( } p< \\
0.01 \text {; values not available) }\end{array}$ \\
\hline & age-matched active females $n=11$ & & Jump height: \\
\hline & & & Dancers: $37.6 \pm 5.5 \mathrm{~cm}$ \\
\hline & & & Controls: $35.9 \pm 3.9 \mathrm{~cm}$ \\
\hline & & & No significant difference \\
\hline & & & \%BF: \\
\hline & & & Dancers: $21.4 \pm 2.8$ \\
\hline & & & Controls: $25.6 \pm 3.7$ \\
\hline & & & Significant difference \\
\hline & & & FFM: \\
\hline & & & Dancers: $42.2 \pm 3.7 \mathrm{~kg}$ \\
\hline & & & Controls: $42.2 \pm 6.6 \mathrm{~kg}$ \\
\hline & & & No significant difference \\
\hline \multirow{8}{*}{$\begin{array}{l}\text { Thomas } 2003 \\
\text { [44] }\end{array}$} & performing dance students & Heel - rises muscular & Number of RPL: \\
\hline & & endurance & Contemporary: $26.4 \pm 3.8$ \\
\hline & & & Ballet: $25.4 \pm 3.7$ \\
\hline & & & Folk: $33.0 \pm 3.64$ \\
\hline & contemporary $n=15$ & & Number of RPkg: \\
\hline & ballet $n=15$ & & Contemporary: $19.1 \pm 3.1$ \\
\hline & folk $n=19$ & & Ballet: $18.9 \pm 3.05$ \\
\hline & other styles $n=41$ & & Folk: $23.0 \pm 3$ \\
\hline \multirow{14}{*}{$\begin{array}{l}\text { Brown et al. } \\
2007 \text { [11] }\end{array}$} & 18 dance students weight training & $\mathrm{F}=1-1.30 \mathrm{~h} / \mathrm{wk}$ & Plyometric training group pre intervention: \\
\hline & $\mathrm{n}=6$ plyometric training $\mathrm{n}=6$ & Intensity- weight & Leg press strength: $183 \pm 30.9 \mathrm{~kg}$ \\
\hline & controls $\mathrm{n}=6$ intervention non $\mathrm{RCT}$ & training $=80 \%$ of 1 & Vertical jump from standing $12.0 \pm 1.2$ in \\
\hline & & $\begin{array}{l}\text { repetition maximum } \\
3 \text { sets of } 6-8 \text { repetitions }\end{array}$ & Aesthetic evaluation - jump height: $3.2 \pm 0.4$ \\
\hline & & Intensity- plyometric $=3$ & Plyometric training group post intervention: \\
\hline & & sets of 8 repetitions of 4 & Leg press strength: $251.5 \pm 39.4 \mathrm{~kg}{ }^{*}$ \\
\hline & & exercises $\mathrm{PD}=12$ weeks & Vertical jump from standing $13.0 \pm 1.0$ in * \\
\hline & & & Aesthetic evaluation - jump height: $3.6 \pm 0.5$ \\
\hline & & & $\begin{array}{l}\text { = significant differences" } \\
\text { Weight training group pre intervention: }\end{array}$ \\
\hline & & & Leg press strength: $214 \pm 61.0 \mathrm{~kg}$ \\
\hline & & & Knee curl strength: $34.8 \pm 4.5 \mathrm{~kg}$ \\
\hline & & & Anaerobic mean power: $340.8 \pm 53.5$ Watts \\
\hline & & & Aesthetic evaluation - feet point: $3.0 \pm 1.2$ \\
\hline & & & Aesthetic evaluation - jump height: $2.8 \pm 1.0$ \\
\hline
\end{tabular}


Table 3 Continued.

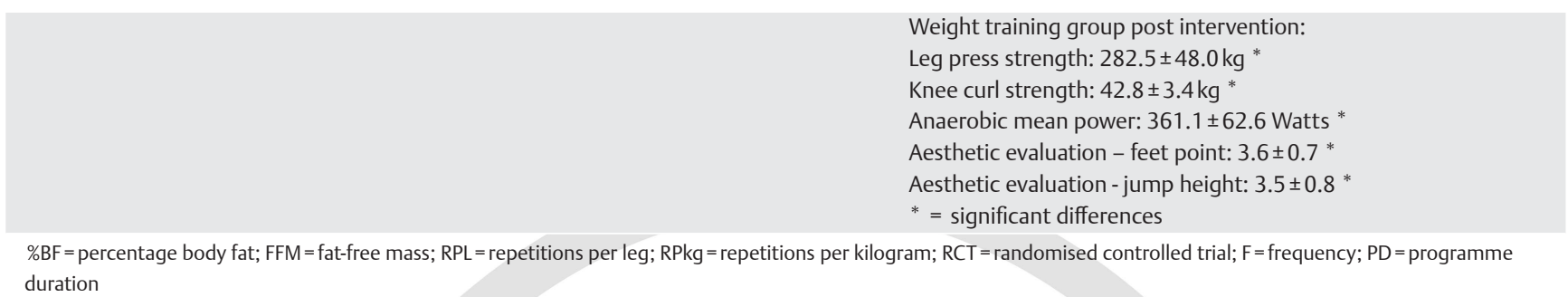

duration

ate between their levels (student and professional), with respect to their fitness, strength and body composition characteristics.

An important aspect which has to be stressed herein, is the link between dance performance and fitness. It has been suggested that although the former has significant "aesthetic" elements, it is the overall fitness of the individual dancer that determines the final outcome [26]. For an optimal stage performance, the dancers aerobic and anaerobic capacities, strength power and endurance as well as their flexibility levels, must be at their peak on the day they are needed. Hence, it is peremptory that dancers, akin to all performing athletes, must adhere to the principles of periodisation and regular evaluation of their fitness levels (e.g. strength, flexibility) via validated laboratory procedures. However, unlike physical fitness and its well defined components [18], the description and quantification of dance performance is less clear, since there are no validated tools that assess full performance. To date research studies have only attempted to quantify and score aspects of dance performance such as overall proficiency, full body involvement, articulation and skills [30]. Therefore, future research in dance science should focus on the development of valid and reliable tools that will enable the accurate assessment and prediction of stage dance performance.

At a professional level, it appears that contemporary dancers demonstrate higher $\mathrm{VO}_{2 \max }$ and \% $\mathrm{BF}$ than ballet. However, student contemporary dancers are equally fit compared to their ballet counterparts and their body composition is also very similar. Comparisons between contemporary, ballet and athletes of other sports are presented in 0 Table 1. It is worth noting that - Table 1 depicts values only for $\mathrm{VO}_{2 \max }$ and body composition of female dancers; the lack of relevant data for professional and student male dancers (either contemporary or ballet), did not allow us to produce a similar table. In addition, the different techniques, equipment and muscular groups do not currently allow for a representative and thorough comparison in strength levels between contemporary dancers with athletes of other sports. Available data, however, showed that muscular strength and power among intermediate dance students, advanced dance students and sedentary individuals do not differ significantly [12] whereas contemporary dancers reported higher scores in muscular endurance compared to ballet dancers [44]. Anaerobic fitness was found to be the least studied component. However, investigating this fitness aspect is indeed important in dance given that actions requiring high power output rely predominantly on the ATP-CP and glycolytic system of energy [5], and dance is characterised by short explosive movements, such as jump series, interspersed between longer periods of rest [52]. Despite this, data from two studies revealed no significant differences in mean anaerobic power between performing and recreational dance students and between various levels of dancers compared to sedentary populations $[12,38]$. However, following supplemental weight training, dancers improved their anaerobic power which also revealed a concomitant improvement in aesthetic evaluation of jump ability [11].

In summary, it appears that both contemporary and ballet dancers have aerobic fitness levels higher to that seen in sedentary individuals [36] and somewhat lower compared to other sports. In addition, muscular strength is higher in professional dancers compared to other athletes [17], however, at a student level no significant differences exist in this fitness component. The reason for this phenomenon is probably the fact that the dance training is not sufficient enough to overload the aerobic/ anaerobic and musculoskeletal systems $[12,26]$ and thus, to produce physiological adaptations that will enhance each individual fitness component. On the other hand, dance specialists and physiologists have to consider how these potential adaptations (gained through supplementary aerobic/strength training) will benefit dance performance since, at least professional dancers, may produce a high quality performance even if their $\mathrm{VO}_{2 \max }$ is lower compared to other sports.

The effectiveness of supplementary aerobic/strength training in order to improve dance performance has been very frequently discussed in published manuscripts (both in trials and reviews) but has only been recently investigated $[11,25]$. One well-methodologically designed study [25] suggests that in students, increases in fitness components result in concomitant beneficial effects in aspects of performance. Nevertheless, the limitations of this study - according to the CONSORT guidelines for RCTs were the method of randomization, the lack of justification for the sample sizes used and finally the lack of presenting the participants' flow diagram. Although the results of this study are in line with the non-randomised study by Brown [11], these studies can only be interpreted as preliminary data and have to be confirmed in future prospective and well-designed studies.

Improvement in individual fitness components may be important for different reasons. In professional dancers, knee extensor and flexor low muscle strength levels have been associated with increased injury severity, expressed as the total time off dance training [27]. The injury recovery process may take longer in dancers with reduced muscular strength, because joints surrounded by weaker soft tissue are subject to more strain due to overexertion, and therefore take longer to recover from the cause of the injury $[31,49]$. Since dance training is not sufficient enough to overload the musculoskeletal systems [12,26], and considering the high injury rates found in dance [47], the implementation of strength training could therefore be recommended as a preventive measure, at least for less strong dancers [24]. In addition, improvements in aerobic/anaerobic capacities and muscular strength have been previously linked to better oxygen transport facilities [4] and enhanced neuromuscular function 
Table 4 Studies primarily investigating body composition characteristics of contemporary dancers.

\begin{tabular}{|c|c|c|c|}
\hline $\begin{array}{l}\text { Author } \\
\text { (reference) }\end{array}$ & Participants & Method & Results \\
\hline $\begin{array}{l}\text { Yannakoulia et al. } \\
2004 \text { [58] }\end{array}$ & $\begin{array}{l}\text { female dance students } \mathrm{n}=37 \\
\text { controls: age- and weight-matched } \\
\text { reference population }\end{array}$ & BMD & $\begin{array}{l}\text { Dancers BMD: } 1.185 \mathrm{~g} / \mathrm{cm} 2 \text { ( } 9 \% \text { higher than } \\
\text { reference values) }\end{array}$ \\
\hline \multirow[t]{4}{*}{$\begin{array}{l}\text { Berlet et al. } \\
2002[9]\end{array}$} & professional female dancers $\mathrm{n}=9$ & $\begin{array}{l}\text { BMD, total regional fat } \\
\text { (\%), TLM }\end{array}$ & $\begin{array}{l}\text { Female dancers: BMD: } 1.18 \pm 0.1 \mathrm{~g} / \mathrm{cm}^{2} \\
\text { Total regional fat: } 13.6 \pm 3.3 \% \text { TLM: } 42.928 \pm 3.4 \mathrm{~g}\end{array}$ \\
\hline & non-dancers $\mathrm{n}=26$ & & $\begin{array}{l}\text { Female controls (weight-matched): } \\
\text { BMD: } 1.19 \pm 0.1 \mathrm{~g} / \mathrm{cm}^{2} \\
\text { Total regional fat: } 9.7 \pm 2.5 \% \text { TLM: } 37.689 \pm 3.4 \mathrm{~g} \\
\text { Significant differences in } \% \mathrm{BF} \text { and TLM }\end{array}$ \\
\hline & professional male dancers $n=6$ & & $\begin{array}{l}\text { Male dancers: BMD: } 1.35 \pm 0.1 \mathrm{~g} / \mathrm{cm}^{2} \\
\text { Total regional fat: } 14.4 . \pm 4.8 \% \text { TLM: } 60.132 \pm 6.8 \mathrm{~g}\end{array}$ \\
\hline & non-dancers $\mathrm{n}=15$ & & $\begin{array}{l}\text { Male controls (weight-matched): } \\
\text { BMD: } 1.25 \pm 0.1 \mathrm{~g} / \mathrm{cm}^{2} \\
\text { Total regional fat: } 24.0 \pm 5.7 \% \text { TLM: } 56.412 \pm 4.6 \mathrm{~g} \\
\text { Sianificant differences in BMD total regional fat }\end{array}$ \\
\hline $\begin{array}{l}\text { Yannakoulia et al. } \\
2000[59]\end{array}$ & elite female dance students $n=42$ & $\begin{array}{l}\text { Investigation of levels of } \\
\text { BMD, FFM, \% BF }\end{array}$ & $\begin{array}{l}\text { Significant differences in BMD, total regional fat } \\
\text { BMD: } 1.180 \pm 0.1 \mathrm{~g} / \mathrm{cm}^{2} \mathrm{FFM}: 42.55 \pm 3.3 \mathrm{~kg} \\
\% \mathrm{BF} \text { (Skinfold): } 21.3 \pm 3.2 \% \mathrm{BF}(\mathrm{DXA}): 19.4 \pm 4.3\end{array}$ \\
\hline $\begin{array}{l}\text { Miletic et al. } \\
2007 \text { [33] }\end{array}$ & $\begin{array}{l}\text { female students with experience in contempo- } \\
\text { rary, ballet and rhythmic gymnastics } n=24 \\
\text { active female students with no previous } \\
\text { experience in aesthetic disciplines } n=21\end{array}$ & $\begin{array}{l}\text { Sum-SF, calf } \\
\text { circumference }\end{array}$ & $\begin{array}{l}\text { Experienced students: Sum-SF: } 28.45 \pm 8.2 \mathrm{~mm} \\
\text { Calf circumference: } 36.42 \pm 2.2 \mathrm{~cm} \\
\text { Non experienced students: Sum-SF: } 23.47 \pm 5.2 \mathrm{~mm} \\
\text { Calf circumference: } 35.98 \pm 2.26 \mathrm{~cm} \\
\text { Significant difference in Sum-SF }\end{array}$ \\
\hline
\end{tabular}

$\mathrm{BMD}=$ bone mineral density; $\mathrm{TLM}=$ Total lean mass; FFM = fat-free mass; \%BF = percentage body fat; DXA = dual- $\mathrm{x}$ ray absorptiometry; Sum-SF = sum of skinfolds

[20], which in turn, affect qualitative elements of physical performance through reduced fatigue $[39,43]$ and injury rates [23]. In conclusion, fitness levels of dancers vary according to different styles and levels and thus future research should distinguish between not only levels of dancers but also styles. It appears that contemporary dancers demonstrate higher maximal oxygen uptake than ballet, while contemporary dance students are equally fit compared to their ballet counterparts and their body composition is also very similar. Similar values were detected in anaerobic fitness, muscular strength/power between various levels of dancers compared to the normal population, whereas contemporary dancers reported higher scores in muscular endurance than ballet counterparts. From the results of this systematic review it appears that the majority of research studies in dance have focused on the assessment of dancers' levels of fitness. In contrast, there is a lack of studies trying to identify an objective assessment of contemporary dance performance. In the two studies investigating the effects of an intervention on dance, two different dance-based tests were used [11,25]; these were the most externally valid to 'dance performance'. However, both tests that were employed to assess aspects of aesthetic competence were not previously validated appropriately, which is a major limitation that should be addressed in similar studies in the future. The data of these preliminary research studies, however, suggest that aerobic and strength training improve overall aesthetic competence, dance technique and aesthetic jump performance of dance students. Further research is needed in order to confirm these preliminary data.

\section{Acknowledgements}

We wish to thank the Arts and Humanities Research Council, U.K., for their funding that made this research possible.

\section{References}

1 Alexander MJL. The physiological characteristics of elite rhythmic sportive gymnasts. J Hum Movement Studies 1989; 17: 49-69

2 Allen $N$, Wyon M. Dance medicine: Artist or athlete? SportEX medicine 2008; 35: 6-9

3 Alméras N, Lemieux S, Bouchard C, Tremblay A. Fat gain in female swimmers. Physiol Behav 1997; 61: 811-817

4 Astrand P, Rodhal K. Textbook of work physiology: physiological bases of exercise. New York: McGraw-Hill International Editions; 1986

5 Baechle TR, Earle RW. Essentials of strength training and conditioning. Champaign, IL: Human Kinetics; 2000

6 Baldari C, Guidetti L. VO2max, ventilatory and anaerobic thresholds in rhythmic gymnasts and young female dancers. J Sports Med Phys Fitness 2001; 41: 177-182

7 Bandyopadhyay A. Anthropometry and body composition in soccer and volleyball players in West Bengal, India. J Physiol Anthropol 2007; 24: 501-505

8 Bennel K, Khan KM, Matthews B, De Gruyter M, Cook E, Holzer K, Wark JD. Hip and ankle range of motion and hip muscle strength in young female ballet dancers and controls. Br J Sports Med 1999; 33: $340-346$

9 Berlet GC, Kiebzak GM, Dandar A, Wooten C, Box JH, Anderson RB, Davis $W H$. Prospective analysis of body composition and sf36 profiles in professional dancers over a 7-month season. Is there a correlation to injury? J Dance Med Sci 2002; 6: 54-61

10 Boileau RA, Mayhew JL, Riner WF, Lussier L. Physiological characteristics of elite middle and long distance runners. Can J Appl Sport Sci 1982; 7: 167-172

11 Brown AC, Wells TJ, Shade ML, Smith DL, Fehling PC. Effects of plyometric training versus traditional weight training on strength, power, and aesthetic jumping ability in female collegiate dancers. J Dance Med Sci 2007; 11: 38-44

12 Chatfield SJ, Byrnes WC, Lally DA, Rowe S. Cross-sectional physiologic profiling of modern dancers. Dance Res J 1990; 22: 13-19

13 Chmelar RD, Schultz BB, Ruhling RO. A physiologic profile comparing levels and styles of female dancers. Phys Sportsmed 1988; 16: 87-96

14 Cohen JL, Segal KR, Witriol IRA, MacArdle WD. Cardiorepiratory responses to ballet exercise and the VO2max of elite ballet dancers. Med Sci Sports Exerc 1982; 14: 212-217

15 Dahlstrom PT, Inasio E, Kaijser L. Physical fitness effort in dancers: a comparison of four major dance styles. Impulse 1996; 4: 193-209

16 Goswami A, Gupta S. Cardiovascular stress and lactate formation during gymnastic routines. J Sports Med Phys Fitness 1998; 38: $317-322$ 
17 Harley YXR, Gibson ASC, Harley EH, Lambert MI, Vaughan CL, Noakes $T D$. Quadriceps strength and jumping efficiency in dancers. J Dance Med Sci 2002; 6: 87-94

18 Heyward VH. Advanced fitness assessment and exercise prescription. Champaign: Illinois: Human Kinetics Publishers Inc.; 2002

19 Hume PA, Hopkins WG, Robinson DM. The predictor of attainment in rhythmic sportive gymnastics. J Sports Med Phys Fitness 1993; 33: 367-377

20 Jones DA, Round JM. Skeletal Muscle in Health and Disease. Manchester: Manchester University Press; 1990

21 Kaufman BA, Warren MP, Dominguez JE, Wang J, Heymsfield SB, Pierson $R N$. Bone density and amenorrhea in ballet dancers are related to a decreased resting metabolic rate and lower leptin levels. J Clin Endocrinol Metab 2002; 87: 2777-2783

22 Kirkendall DT, Calabrese LH. Physiological aspects of dance. Clin Sports Med 1983; 2 (3): 525-536

23 Knapic JJ, Jones BH, Bauman CL, Harris JM. Strength, flexibility and athletic injuries. Sports Med 1992; 14: 277-288

24 Koutedakis Y, Cross V, Sharp NCC. The effects of strength training in male ballet dancers. Impulse 1996; 4: 210-219

25 Koutedakis Y, Hukam H, Metsios G, Nevill A, Giakas G, Jamurtas A, Myszkewycz $L$. The effects of three months of aerobic and strength training on selected performance and fitness-related parameters in modern dance students. J Strength Cond Res 2007; 21: 808-812

26 Koutedakis $Y$, Jamurtas $A$. The dancer as a performing athlete: physiological considerations. Sports Med 2004; 34: 651-661

27 Koutedakis Y, Khaloula M, Pacy PJ, Murphy M, Dunbar GMJ. Thigh peak torques and lower-body injuries in dancers. J Dance Med Sci 1997; 1: $12-15$

28 Koutedakis $Y$, Sharp C. The fit and healthy dancer. Chichester: John Wiley and Sons 1999

29 Krasnow D, Kabbani M. Dance science research and the modern dancer. Med Probl Perform Ar 1999; 14: 16-20

30 Krasnow DH, Chatfield SJ, Barr S, Jensen JL, Dufek JS. Imagery and conditioning practices for dancers. Dance Res J 1997; 29: 43-65

31 Kumar S, Tomic E. Theories of musculoskeletal injury causation. Ergonomics 2001 ; 44: 17-47

32 Malousaris GG, Bergeles NK, Barzouka KG, Bayios IA, Nassis GP, Koskolou $M D$. Somatotype, size and body composition of competitive female volleyball players. J Sci Med Sport 2008; 11: 337-344

33 Miletic $D$, Sekulic $D$, Ostojic $L$. Body physique and prior training experience as determinants of SEFIP score for university dancers. Med Probl Perform Ar 2007; 22: 110-115

34 Mitsuzono R, Ube M. Effects of endurance training on blood lipid profiles in adolescent female distance runners. Kurume Med J 2006; 53 : 29-35

35 Mladenoviæ I. Developing characteristics and functional abilities of top female football players. Facta Universitatis - Medicine and Biology 2005; 12: 97-99

36 Novak LP, Magill LA, Schutte JE. Maximal oxygen intake and body composition of female dancers. Eur J Appl Physiol 1978; 39: 277-282

37 O'Mailia SP, Scharff-Olson M, Williford HN. Activity monitors and dance-based exercise. Estimating caloric expenditure. J Dance Med Sci 2002; 6: 50-53

38 Padfield JA, Eisenman PA, Luetkemeier MJ, Fitt SS. Physiological profiles of performing and recreational early adolescent female dancers. Pediatr Exerc Sci 1993; 5: 51-59

39 Petibois C, Cazorla G, Poortmans JR, Deleris G. Biochemical aspects of overtraining in endurance sports: A review. Sports Med 2002; 32: $867-878$
40 Redding E, Wyon MA. Strengths and weaknesses of current methods for evaluating the aerobic power of dancers. J Dance Med Sci 2003; 7: $10-16$

41 Reid DC. Prevention of hip and knee injuries in ballet dancers. Sports Med 1988; 6: 295-307

42 Rodriguez FA. Maximal oxygen uptake and cardiorespiratory response to maximal $400 \mathrm{~m}$ free swimming, running and cycling tests in competitive swimmers. J Sports Med Phys Fitness 2000; 40: 87-95

43 Romer LM, MacConnel AK, Jones DA. Effects of inspiratory mucle training upon recovery time during high intensity, repetitive sprint activity. Int J Sport Med 2002; 23: 353-360

44 Thomas KS. Functional eleve' performance as it applies to heel-rises in performance-level collegiate dancers. J Dance Med Sci 2003; 7: $115-120$

45 Tsunawake N, Tahara Y, Moji K, Muraki S, Minowa K, Yukawa K. Body composition and physical fitness of female volleyball and basketball players of the Japan inter-high school championship teams. J Physiol Anthropol Appl Human Sci 2003; 22: 195-201

46 Van Marken Lichtenbelt WD, Fogelholm M, Ottenheijm R, Westerterp $K R$. Physical activity, body composition and bone density in ballet dancers. Br J Nutr 1995; 74: 439-451

47 Weigert BJ, Erickson M. Incidence of injuries in female university-level modern dancers and the effectiveness of a screening program in altering injury patterns. Med Probl Perform Ar 2007; 22: 52-57

48 Weimann E. Gender-related differences in elite gymnasts: the female athlete triad. J Appl Physiol 2002; 92: 2146-2152

49 Weiss DS, Zlatkowski M. Rehabilitation of dance injuries to the shoulder, lumbar spine, pelvis and hip. Orthop Phys Ther Clin North Am 1996; 5: 477-496

50 White SB, Philpot A, Green A, Bemben MG. Physiological comparison between female university ballet and modern dance students. J Dance Med Sci 2004; 8: 5-10

51 Whyte GP, George K, Redding E, Wilson M, Lane A, Firooz S. Electrocardiography and echocardiography findings in contemporary dancers. Dance Med Sci 2003; 7: 91-95

52 Wyon MA. Cardiorespiratory training for dancers. J Dance Med Sci 2005; 9: 7-12

53 Wyon MA. Testing an aesthetic athlete: contemporary dance and classic ballet dancers. In: Winter E, Jones AM, Davison RCR, Bromley PD, Mercer TH, eds. Sport and Exercise Physiology testing guidelines. London: Routledge; 2007; 242-262

54 Wyon MA, Abt G, Redding E, Head A, Sharp NCC. Oxygen uptake during modern dance class, rehearsal, and performance. J Strength Cond Res 2004; 18: 646-649

55 Wyon MA, Head A, Sharp C, Redding E. The cardiorespiratory responses to modern dance class. Differences between university, graduate and professional classes. J Dance Med Sci 2002; 6: 41-45

56 Wyon MA, Redding E. Physiological monitoring of cardiorespiratory adaptations during rehearsal and performance of contemporary dance. J Strength Cond Res 2005; 19: 611-614

57 Wyon MA, Redding E, Abt G, Head A, Sharp NC. Development, reliability, and validity of a multistage dance specific aerobic fitness test (DAFT). J Dance Med Sci 2003; 7: 80-84

58 Yannakoulia M, Keramopoulos A, Matalas AL. Bone mineral density in young active females: the case of dancers. Int J Sport Nutr Exerc Metab 2004; 14: 285-297

59 Yannakoulia M, Keramopoulos A, Tsakalakos N, Matalas AL. Body composition in dancers: the bioelectrical impedance method. Med Sci Sports Exerc 2000; 32: 228-234

\section{Erratum}

M. Angioi, G. Metsios, Y. Koutedakis, M. A. Wyon

Fitness in Contemporary Dance: A Systematic Review DOI 10.1055/s-0029-1202821 / Published online: March 19, 2009; Int J Sports Med 2009; 30: 475-484

An error has occurred in this article. The name of one of the authors should be "G. S. Metsios" instead of "G. Metsios". 